\title{
ORNL actinide materials and a new detection system for superheavy nuclei
}

\author{
Krzysztof P. Rykaczewski ${ }^{1, \mathrm{a}}$, James B. Roberto ${ }^{1}$, Nathan T. Brewer ${ }^{1,2}$, \\ and Vladimir K. Utyonkov ${ }^{3}$ \\ ${ }^{1}$ Oak Ridge National Laboratory, Physics Division, Oak Ridge, TN 37831, USA \\ ${ }^{2}$ University of Tennessee, Department of Physics and Astronomy, Knoxville, TN 37996, USA \\ ${ }^{3}$ Joint Institute for Nuclear Research, RU-141980 Dubna, Russian Federation
}

\begin{abstract}
The actinide resources and production capabilities at Oak Ridge National Laboratory (ORNL) are reviewed, including potential electromagnetic separation of rare radioactive materials. The first experiments at the Dubna Gas Filled Recoil Separator (DGFRS) with a new digital detection system developed at ORNL and University of Tennessee Knoxville (UTK) are presented. These studies used ${ }^{240} \mathrm{Pu}$ material provided by ORNL and mixed-Cf targets made at ORNL. The proposal to use an enriched ${ }^{251} \mathrm{Cf}$ target and a large dose of ${ }^{58} \mathrm{Fe}$ beam to reach the $N=184$ shell closure and to observe new elements with $Z=124,122$ and 120 is discussed.
\end{abstract}

\section{ORNL actinide resources}

Actinide materials originating from the ORNL's High Flux Isotope Reactor (HFIR) and Radiochemical Engineering Development Center (REDC) have been used in experiments identifying nine superheavy elements, from $Z=104$ to 106 and from $Z=113$ to 118 . The relatively high power of HFIR combined with its flux trap design provide an intense steady-state neutron flux of $2.4 * 10^{15}$ neutrons $/ \mathrm{s} / \mathrm{cm}^{2}$. This very high neutron flux is essential for actinide production. The adjacent REDC operates several hot cells capable of handling highly radioactive materials from HFIR. REDC provides unique capabilities for the efficient processing and separation of actinide materials including $Z=98{ }^{252} \mathrm{Cf}$ with a half-life of 2.6 years. ${ }^{252} \mathrm{Cf}$ is produced in HFIR in hundred milligram quantities from Am and $\mathrm{Cm}$ feedstock material, mostly recoverable after production and separation cycles lasting from several months to two years. $Z=97{ }^{249} \mathrm{Bk}$ can be obtained as a bi-product of ${ }^{252} \mathrm{Cf}$ production campaigns [1]. Currently, approximately $10 \mathrm{mg}$ of ${ }^{249} \mathrm{Bk}$ can be separated after a typical ${ }^{252} \mathrm{Cf}$ campaign. Increasing the production of ${ }^{249} \mathrm{Bk}$ requires a dedicated irradiation in HFIR using a suppressed thermal neutron flux tailored for ${ }^{249} \mathrm{Bk}$ production [2]. Such an approach can increase ${ }^{249} \mathrm{Bk}$ production by factors of two or more but is more costly since it is not compatible with standard ${ }^{252} \mathrm{Cf}$ production campaigns. Actinide materials valuable for research on superheavy nuclei include highly enriched ${ }^{237} \mathrm{~Np},{ }^{242} \mathrm{Pu},{ }^{241,243} \mathrm{Am},{ }^{244} \mathrm{Cm}$, and ${ }^{248} \mathrm{Cm}$. Up to gram quantities of these isotopes are currently in actinide inventories at ORNL, with the exception of ${ }^{248} \mathrm{Cm}$ where the current inventory is $80 \mathrm{mg}$. REDC is currently recovering about $10 \mathrm{mg} /$ year of highly enriched ${ }^{248} \mathrm{Cm}$ from old ${ }^{252} \mathrm{Cf}$ sources. In addition, about $15 \mathrm{mg}$ of mixed-Cf material, containing longer-lived ${ }^{249} \mathrm{Cf}(50 \%),{ }^{250} \mathrm{Cf}(15 \%)$, and

\footnotetext{
${ }^{a}$ Corresponding author: rykaczewskik@ornl.gov
}

(C) The Authors, published by EDP Sciences. This is an Open Access article distributed under the terms of the Creative Commons Attribution License 4.0 (http://creativecommons.org/licenses/by/4.0/). 

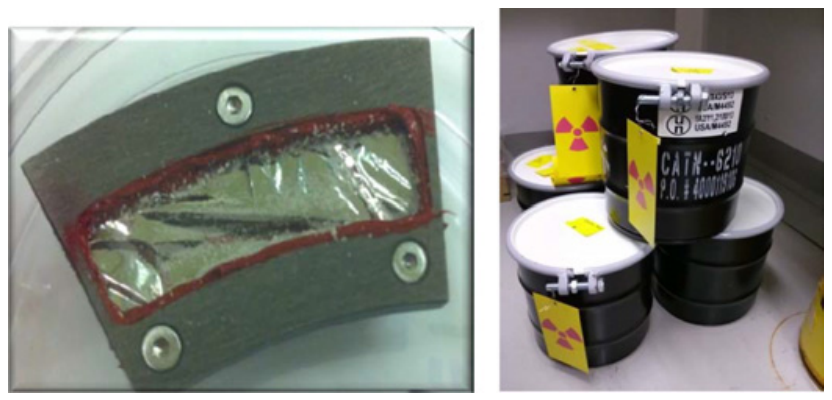

Figure 1. Left panel: One of the mixed-Cf target sectors electrodeposited on Ti foils at ORNL's REDC. Right panel: Twelve target sectors with required shielding arriving at JINR Dubna early 2015.

${ }^{251} \mathrm{Cf}(35 \%)$, has recently been recovered from these old sources. In 2015, twelve target sectors containing mixed-Cf, with a typical thickness of about $0.33 \mathrm{mg} / \mathrm{cm}^{2}$ and $3-\mathrm{cm}^{2}$ area, were electrodeposited at REDC on Ti-foils for a planned experiment at JINR to search for heavier isotopes of element 118 (see Fig. 1). The total content of ${ }^{252} \mathrm{Cf}$ in all sectors was below two micrograms. However, with a high percentage of ${ }^{250} \mathrm{Cf}$ (half-life $T_{1 / 2}=13$ years), the neutron emission from the target material is still quite high, at the level of $2 * 10^{7}$ neutrons per second into full solid angle.

The isotope ${ }^{251} \mathrm{Cf}\left(T_{1 / 2}=898\right.$ years) is the most valuable component of the mixed-Cf target enabling the possible synthesis of new $Z=118$ isotopes, two neutrons closer to the predicted $N=184$ shell closure. Technically, it would be possible to mass separate tens of milligrams of ${ }^{251} \mathrm{Cf}$ suitable for a target wheel containing nearly $100 \%$ enriched ${ }^{251} \mathrm{Cf}$. This would require an efficient electromagnetic separator dedicated to radioactive materials. A lower current prototype separator is currently operating at ORNL to separate stable isotopes. A similar separator optimized for actinide materials would be more than adequate for the needs of the superheavy element research community.

\section{Experiments with the ORNL/UTK digital detection system at the DGFRS}

A new detection system for superheavy nuclei has been designed, built and commissioned at ORNL. The concept follows the experience gained during discovery research on proton and $\alpha$ emitters at the Recoil Mass Separator at ORNL's Holifield Radioactive Ion Beam Facility. The new detector system uses a very large Si-wafer, with $128 \mathrm{~mm}$ by $48 \mathrm{~mm}$ active area, processed to work as a Double-sided Silicon Strip Detector (DSSD) by Micron Semiconductor (BB-17 type, 300 microns thick). This detector is installed at the final focus of the DGFRS, to detect implanted nuclei and observe related $\alpha$ and fission decays. It has a matching size silicon single veto detector and six single detectors of $120 \mathrm{~mm}$ by $60 \mathrm{~mm}$ area acting as an escape-catching silicon-detector box. This system has replaced the position sensitive silicon detectors used earlier at the DGFRS for studies of superheavy nuclei. All silicon detectors and two Dubna recoil transmission detectors Multi-Wire Proportional Chambers (MWPCs) are operated using Pixie 16 digital signal processing electronics manufactured by XIA [3], in parallel to the local analogue data acquisition system. The digital data acquisition system was developed at the UTK's Digital Pulse Processing Laboratory, by R. Grzywacz and collaborators [4], and at ORNL, with contributions by K. Miernik (Oak Ridge/Warsaw) and N. Brewer (Oak Ridge). All single pulses are analysed on board the Pixie-16 module, while on-board recognized pile-up signals occurring within ten microseconds are recorded as digital 


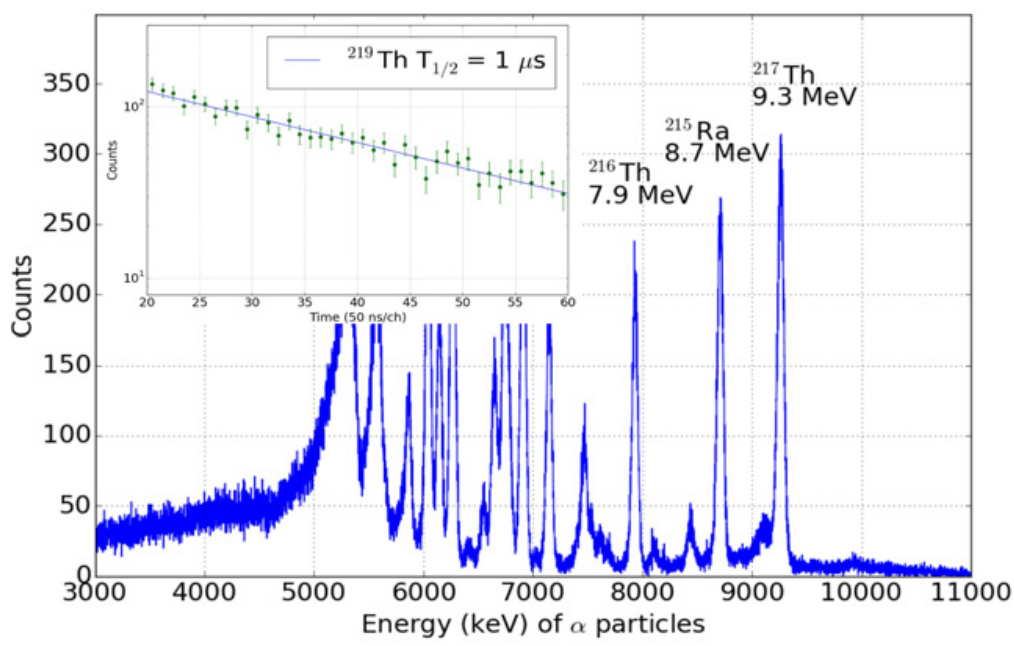

Figure 2. Energy spectrum of $\alpha$ particles emitted from the fusion-evaporation products of the ${ }^{48} \mathrm{Ca}+{ }^{\text {nat }} \mathrm{Yb}$ reaction and measured using the new digital detection system at the DGFRS. The inset shows the decay pattern of $\alpha$ particles emitted from a $1-\mu$ s activity of ${ }^{219} \mathrm{Th}$ derived from collected traces of the DSSD preamplifier signals.

pulse images, with 10-ns time resolution, for initial on-line and further off-line analysis. An example of the capability of the new digital detection system is illustrated in Fig. 2.

The reaction between a $Z=70$ natural ytterbium target and ${ }^{48} \mathrm{Ca}$ ions was used to calibrate the $\alpha$-energy scale of the silicon detectors. Short-lived $Z=90$ thorium activities were produced and implanted in the DSSD, and their decay was recorded. This includes the direct observation of the implantation and decay of ${ }^{219} \mathrm{Th}$ activity having a $1-\mu$ s half-life. The ${ }^{219}$ Th decay curve was obtained through the analysis of recoil-decay signal traces. For the first time, the recoil implantation followed by a decay of such a short-lived activity was directly identified at the DGFRS.

\subsection{Fusion-evaporation reactions between a ${ }^{48} \mathrm{Ca}$ beam and ${ }^{239,240} \mathrm{Pu}$ targets}

The first experiment aiming at new superheavy nuclei and using the new digital detection system was performed at the DGFRS with a ${ }^{48} \mathrm{Ca}$ beam irradiating ${ }^{239} \mathrm{Pu}$ and ${ }^{240} \mathrm{Pu}$ targets (the latter material provided from ORNL). The spectroscopy results include the observation of three decay chains of ${ }^{285} \mathrm{Fl}$ including the previously not detected full energy of the first $\alpha$ particle and evidence for the new even-even isotope ${ }^{284} \mathrm{Fl}$ undergoing fast spontaneous fission [5]. The low cross sections detected in the experiments with ${ }^{239,240} \mathrm{Pu}$ targets compared to the larger values observed with ${ }^{242,244} \mathrm{Pu}$ targets indicate that the west shore of the Island of Stability has been identified, as illustrated in Fig. 3.

\subsection{Irradiation of a mixed-Cf target with a ${ }^{48} \mathrm{Ca}$ beam}

The irradiation of the ORNL mixed-Cf target with a ${ }^{48} \mathrm{Ca}$ beam started at the DGFRS at the beginning of October 2015. After nine days of bombardment at about 0.5 particle- $\mu \mathrm{A}$, a decay chain of the isotope ${ }^{294} 118$ was observed through both analogue and digital data acquisitions [6]. The observed decay properties were close to the previously identified four chains of this only known isotope of the heaviest known atomic element. Two $\alpha$ decays followed by a 


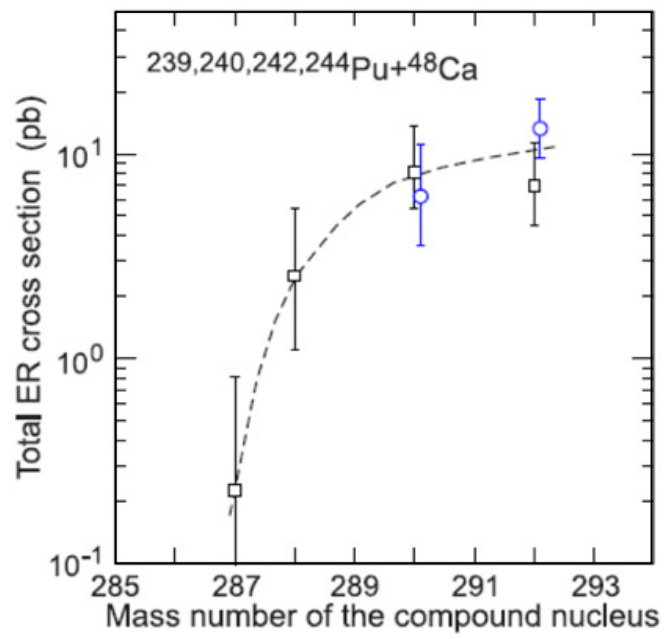

Figure 3. The cross section pattern observed for the $Z=114 \mathrm{Fl}$ isotopes produced using a ${ }^{48} \mathrm{Ca}$ beam and four Pu targets, with mass $A$ ranging from 239 to 244 (figure courtesy of Physical Review C). The dramatic drop of cross-section values was interpreted as being caused by the reduction of fission barriers with decreasing neutron number of the Fl compound nucleus [5].

spontaneous fission of ${ }^{286} \mathrm{Fl}$ were detected. The experiment continued with slowly increasing beam current, up to 0.7 particle- $\mu \mathrm{A}$. However, frequent checks of the target performance, through the observation of $\alpha$ particles emitted from the target, began to show signs of target thickness increase, i.e., of a build-up of an extra layer of material at the target surface. Optical inspection of the targets indeed revealed a layer of material covering the electrodeposited mixed-Cf material. We are in the process of returning the mixed-Cf target to ORNL, for assessing the problem and refurbishing the target sectors.

\section{Towards the predicted neutron shell closure at $N=184$ and new elements}

The properties of $\alpha$ decays for nuclei produced in the reactions between radioactive actinide targets and a ${ }^{48} \mathrm{Ca}$ beam point clearly to the large enhancement of stability with increasing neutron number. See, e.g., reference [7]. The largest neutron number reached so far is $N=177$, in ${ }^{293} \mathrm{Lv}$ and ${ }^{294} 117$ nuclei. While the spectroscopic evidence is in agreement with a predicted shell closure at $N=184$, the experiment is far from this neutron number. However, use of heavier and more intense beams and the heaviest actinide targets might bring us closer to $N=184$. For example, the reaction between a ${ }^{58} \mathrm{Fe}$ beam and a ${ }^{251} \mathrm{Cf}$ target could create the compound nucleus ${ }^{309} 124$ in an excited state. The isotope ${ }^{309} 124$ has $N=185$ neutrons, i.e., one neutron added to the predicted spherical magic core. At known parts of the Segre chart such "core plus one particle" nuclei have a relatively small binding energy for this valence nucleon. One can expect that the evaporation of the $185^{\text {th }}$ neutron is highly probable. The resulting one-neutron evaporation product, the isotope ${ }^{308} 124$ with $N=184$ neutrons might have stability against particle evaporation and radioactive decay enhanced by its single- or even doubly-magic character. The decay chain starting from the ${ }^{308} 124$ activity would reach the known ${ }^{292} \mathrm{Lv}$ isotope after four $\alpha$ decays. The subsequent two $\alpha$ particles of known properties will populate ${ }^{284} \mathrm{Cn}$ which undergoes spontaneous fission with about 90-ms half-life. It means there are several known spectroscopic signatures allowing 
us to identify the decay of the new nucleus ${ }^{308} 124$. The observation of the one-neutron evaporation channel in the ${ }^{58} \mathrm{Fe}+{ }^{251} \mathrm{Cf}$ reaction would constitute a revival of the "cold fusion" approach in the search for superheavy nuclei. Of course, the qualitative analysis presented here assumes that $N=184$ is a good magic number for a new element with high atomic number, $Z=124$. The HFB calculations summarized recently [8] support the magic character of $N=184$ at $Z=124$ and predict a very large fission barrier in this region of nuclei, of the order of $10 \mathrm{MeV}$. However, microscopic-macroscopic calculations [8] point to rather small fission barriers for the nuclei discussed, closer to $3 \mathrm{MeV}$, indicating that the magic character of $N=184$ does not hold for such superheavy elements. As one can expect, the cross-section estimates vary dramatically with fission barrier parameter values. The very preliminary and rough estimates by Krystyna Wilczyńska indicate that the production yield for the $x n$-evaporation channels might be within experimental reach with the fission barrier parameter even slightly below $5 \mathrm{MeV}$ [9].

However, the experimental attempt to identify the nucleus ${ }^{308} 124$ and following $\alpha$ decay products ${ }^{304} 122$ and ${ }^{300} 120$, i.e., three new elements including a $N=184$ isotone, will be much better justified if an enriched ${ }^{251} \mathrm{Cf}$ target will be available. Therefore, having the actinide separation capability together with a new generation of accelerator laboratories can elevate superheavy element science from our current capabilities, for instance ${ }^{294} 118$, toward further discoveries including several new elements and the magic ${ }^{308} 124$ nucleus.

This research was supported by the U.S. Department of Energy (DOE) Office of Science, Office of Nuclear Physics, Isotope Development and Production for Research and Applications Program, under contract DE-AC05-00OR22725 with UT-Battelle, LLC. We are grateful to the ORNL Radiochemical Engineering Development Center and High Flux Isotope Reactor, a DOE Office of Science, Basic Energy Sciences User Facility, for their support in the production and chemical separation of the actinide materials. We also thank our many collaborators at the Flerov Laboratory of Nuclear Reactions (JINR, Dubna, Russia), Lawrence Livermore National Laboratory, Vanderbilt University, and the University of Tennessee-Knoxville, without whom this research would not have been possible.

\section{References}

[1] J.B. Roberto et al., Nucl. Phys. A944, 99 (2015).

[2] R. Boll et al., Super Heavy Element Target Material Needs and Production at ORNL, unpublished ORNL report, April 2016.

[3] http://xia.com

[4] R.K. Grzywacz et al., Nucl. Instr. Meth. B261, 1103 (2007).

[5] V. K. Utyonkov et al., Phys. Rev. C 92, 034609 (2015).

[6] N.T. Brewer et al., to be published.

[7] V. K. Utyonkov et al., ibidem.

[8] A. Baran et al., Nucl. Phys. A944, 442 (2015).

[9] K. Wilczyńska, private communication (2016). 